\title{
Flat-pack, rapid production powered air purifying respirators for Low-Resource Settings amid the SARS-CoV-2 pandemic
}

Antonio De Grazia ${ }^{1 *}$, Laura E. Diment ${ }^{1}$, Constantinos Regas ${ }^{2}$, Sebastian Rosini ${ }^{1}$ Diana J. GarayBaquero ${ }^{2}$, Nicholas W. Hale ${ }^{1}$, Roeland Mingels ${ }^{4}$, Philip Mattinsley ${ }^{5}$, Alexander S. Dickinson ${ }^{1,6}$, Paul T. Elkington $^{2,3,6}$, and Mark N. Mavrogordato ${ }^{1 *}$.

\footnotetext{
${ }^{1}$ Mechanical Engineering Department, Faculty of Engineering \& Physical Sciences, University of Southampton, UK;

${ }^{2}$ School of Clinical and Experimental Sciences, Faculty of Medicine, University of Southampton, UK;

${ }^{3}$ NIHR Biomedical Research Centre, University Hospital Southampton NHS Foundation Trust, Southampton, UK;

${ }^{4}$ School of Electronics \& Computer Science, Faculty of Engineering \& Physical Sciences, University of Southampton, UK.

${ }^{5}$ Jaguar Land Rover Special Vehicle Operations, Fen End, UK;

${ }^{6}$ Institute for Life Sciences, University of Southampton, UK;

*Corresponding authors: A.DeGrazia@soton.ac.uk; mnm100@soton.ac.uk.
}

Additional data (CADs and videos) has been made openly available from the University of Southampton repository at https://doi.org/10.5258/SOTON/D1582.

Please see Warning and Disclaimer at the end of this document.

\begin{abstract}
Healthcare workers are at high risk of catching SARS-CoV-2 because of their regular interaction with patients with the disease. In low-resource settings, the ratio of healthcare workers to the whole population is lower than in high income countries, and there is often limited access to personal protective equipment (PPE). Illness or death of healthcare workers will, therefore, have a disproportionate impact in these settings, so it is particularly important to find ways to protect them.

To protect against airborne infection in healthcare settings, PPE recommendations typically include filtering facemask respirators or powered air purifying respirators (PAPR). The former, passively filter inhaled air. They are small, noiseless and do not require a power supply, but they are single-use, presenting manufacturing and supply issues. Fit testing is crucial, and many users find them difficult to tolerate, due to breathing resistance and elevated humidity. There is also the potential for contamination due to the exposed face. PAPRs are re-usable devices that may last for months and
\end{abstract}


provide airflow through a filter from a battery-powered blower unit to a hood or helmet which covers the face. This creates a positive pressure in the hood or helmet that enables the wearer to breathe filtered air easily, without requiring an air-tight fit needed for standard face masks. This is reported to be more comfortable and provides better protection for the face from droplets and splashes, and infection by self-contact with the hands. PAPRs have typically been expensive, bulky and not readily available or easy to ship to low-resource settings.

Although the design presented here has not been through any form of regulatory approval, the aim of this paper is to share ideas and offer possible solutions to other groups around the World who may be thinking of manufacturing a low-cost, reusable PAPR. The design is novel because it uses readily available materials, scalable manufacturing processes, and it may be shipped flat-packed and easily assembled. This offers an option for manufacturing in low-resource settings and for shipping in bulk. This paper provides the CAD designs that can be fabricated using a laser cutter.

\section{INTRODUCTION}

\section{The disease and its risks for low-resource regions}

SARS-CoV-2 was classed as a global pandemic by the World Health Organization (WHO) on $11^{\text {th }}$ March 2020 [1][2]. Infection results in the clinical disease COVID-19, and can cause severe acute respiratory syndrome. Early in the pandemic, the healthcare systems of many high-income countries were at capacity and close to being overwhelmed, and the potential health, social and economic impacts of the pandemic on low-resource settings with lesser infrastructure and healthcare availability could be devastating and long-lasting [3][4]. In low-resource settings there is a greater risk that personal protective equipment (PPE) has limited availability, and it is harder for individuals to self-isolate because many of these countries have high population density, larger household sizes and less support for people who lose their jobs or need time off work [5][6][7]. The ratio of healthcare workers to the whole population is lower than in high income countries, and therefore sickness of healthcare workers can have a greater effect [8].

Staff in healthcare settings, including medical staff, allied health professionals, administrators and cleaners, have a high likelihood of catching SARS-CoV-2 because of their regular interaction with patients with the disease [9], and recurrent exposure may be associated with increased mortality [10]. Access to effective PPE for healthcare staff is vital, to minimise the risks of catching symptomatic Covid-19 or asymptomatic onward transmission to patients, colleagues or families [11].

\section{SARS-CoV-2 transmission routes}

The disease primarily spreads through droplet transmission when an infected person exhales, coughs, sneezes, or talks, and a susceptible person inhales aerosol droplets. People can also become infected by touching surfaces that have the virus on them, then touching their eyes, nose or mouth [12]. The SARS-CoV-2 virus is approximately in $50-200 \mathrm{~nm}$ in diameter [13]. However, it is not commonly transmitted as individual virions. It is transmitted, to varying degrees, as aerosol droplet nuclei (smaller than $5 \mu \mathrm{m}$ diameter), respiratory droplets (5-10 $\mu \mathrm{m}$ diameter) and through fomites (via infected surfaces), with the virus coming into contact with the body's mucosal surfaces and airways through touch or re-aerosolisation [14]. Virus remains viable in fomites for varying times, from hours to days depending on the substrate, and lingers as aerosol, with a half-life of 1.2 hours [15]. SARS-CoV-2 has been found to be present in faecal samples [16], and in toilets which have been used by COVID-19 patients or where healthcare workers changed out of their personal protective 
equipment [17]. However, the presence of viral RNA does not necessarily mean that viable virus is present, so the faecal-oral/inhalation route has neither been confirmed nor ruled out.

\section{Current prevention measures}

For prevention of transmission, the WHO advises cleaning hands frequently with soap and water or alcohol-based hand sanitiser, and avoiding contact with people coughing and sneezing [18]. Nevertheless, in countries where access to clean water is limited, even this basic precaution poses challenges [19]. A UNICEF and WHO joint study showed that $40 \%$ of health care facilities are not equipped to practice hand hygiene at point of care, and 4 in 10 households do not have soap and water on premises. Many governments have also advised people to wear face coverings over the nose and mouth when outdoors or meeting other people, to try to reduce the rate of infection [20], and this may reduce availability of PPE for healthcare workers.

\section{Types of PPE: N95/FFP2 face coverings and PAPRs, advantages and disadvantages}

To protect against airborne infection for higher risk aerosol-generating procedures (AGPS) in healthcare settings, PPE recommendations typically include two types of filtering devices: N95/FFP2 respirators (or N99/FFP3 as recommended in the UK) and powered air purifying respirators (PAPR) [21] (see Table 1 for summary of common masks and respirators). The former passively filter inhaled air, requiring removal of $94 \%$ of aerosol particles (smaller than $5 \mu \mathrm{m}$ in diameter) and droplets (5 $\mu \mathrm{m}$ to $50 \mu \mathrm{m}$ ), while allowing for stethoscopes to be used, being noiseless and not requiring power supplies. However, these are recommended for single-use, presenting significant manufacturing, supply and logistic issues, and fit testing is crucial for their optimal use and efficacy. Many users find them difficult to tolerate, due to breathing resistance and moisture formation, and there is a potential for contamination due to the exposed face [21] [22]. On the other hand, PAPRs are reusable devices that may last for months and provide airflow through a filter from a battery-powered blower unit to a hood or helmet which covers face. This creates a positive pressure in the hood or helmet that enables the wearer to breathe filtered air easily, without requiring an air-tight fit needed for standard face coverings. This is reported to be more comfortable and provides better protection for the face from droplets and splashes, and infection by self-contact with the hands [23].

PAPRs contain filters that are usually housed as cartridges and can be interchanged according to the size and type of infection source. A typical filter used in PAPR is the high-efficiency particulate air (HEPA) filter [24], which can filter at least $99.97 \%$ of particles $0.3 \mu \mathrm{m}$ in diameter, meaning that a HEPA filter-equipped PAPR should provide greater protection than most N95/FFP2 respirators. PAPRs may be more inclusive, as they do not necessitate fit testing, and have other advantages, such as benefitting people with hearing impairment who lip read, and improving communication, as patients can see doctors' and nurses' faces [25] [26]. Additionally, PAPRs are easier to wear for a long period of time, because fresh air flows on the user's face and the avoidance of contact with skin on the face might avoid the soft tissue discomfort and skin damage experienced in FFPR use. However, they can be bulkier, noisier, and they require a power supply [21] [27], so they may not always be more usable and inclusive than mask-type respirators. Reusing fabric face coverings is not advisable because SARS-CoV-2 virus survives for a long time on face covering material, so some organisations recommend that they are disposed of after every use, in a hospital context above all because not all of them are fit-tested meaning that there is an airflow leakage around the masks. However, cloth masks help protecting from large droplets especially when composed of different fabric layers [28] [29]. Overall, PAPRs offer better potential protection against respiratory pathogens, they can be cleaned and reused, and their filters can be substituted easily and safely [30] [31]. 
Technical specifications for personal respirators for health-care workers are summarised in a recent work by Elkington et al. [32].

Table 1 - Summary of common types of face coverings and respirators. Filter efficiency, advantages and disadvantages are described for surgical masks, FFP type medical masks and air purifying respirators.

\begin{tabular}{|c|c|c|c|c|}
\hline & Price Scale & \multicolumn{2}{|c|}{ Protection } & Limitations \\
\hline Surgical mask & s & \multicolumn{2}{|c|}{$\begin{array}{l}\text { Surgical masks resistant to fluids protect against large droplets, splashes, or } \\
\text { sprays of } \\
\text { bodily or other hazardous fluids [33]. } \\
\begin{array}{l}\text { Typically FFP2 (Filtering Face Piece - Class 2) protection, but without a tight fit to } \\
\text { the face. }\end{array}\end{array}$} &  \\
\hline FFP1 Medical mask & \multirow{3}{*}{ ss } & \multirow{3}{*}{$\begin{array}{l}\text { The duration of use is dependent on } \\
\text { the environment conditions and } \\
\text { individual factors. These masks } \\
\text { should be disposed of appropriately } \\
\text { after removal [35. } \\
\text { Medical masks work properly when } \\
\text { held tightly at user's face. }\end{array}$} & $\begin{array}{l}\text { FFP1 (Filtering Face Piece - Class 1) } \\
\text { provides protection against low level, } \\
\text { non-hazardous dusts and oil or water } \\
\text { based mists, typicially found during hand } \\
\text { sanding and basic DIY activities [36]. }\end{array}$ & $\begin{array}{l}\text { Fit test is required [37]; } \\
\text { Only suitable for protection against irrtitating, not } \\
\text { harmful substances. It dees not protect against SARS- } \\
\text { CoV-2. } \\
\text { Minimum filter efficiency of } 80 \% \text { [38]. } \\
\text { It must be worn along with eye protection in cases of } \\
\text { AGPS [39]. }\end{array}$ \\
\hline FFP2/N95 Medical mask & & & $\begin{array}{l}\text { FFP2 (FFitering Face Piece - Class 2) } \\
\text { protects against solid and liquid } \\
\text { irritating a erosols [361. Minimum fiter } \\
\text { efficiency of } 94 \%[38] \text {. This is equivalent } \\
\text { to USA approved N95 respirators [40]. }\end{array}$ & $\begin{array}{l}\text { Fit test is required [37]; } \\
\text { It must be worn along with eye protection in cases of } \\
\text { AGPS [39]. }\end{array}$ \\
\hline FFP3 Medical mask & & & 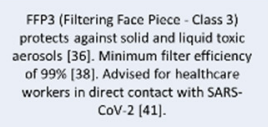 & $\begin{array}{l}\text { Fit test is required } 137] \text {; } \\
\text { It must be worn along with eye protection in cases of } \\
\text { AGPs }[39] \text {. }\end{array}$ \\
\hline $\begin{array}{l}\text { Air Purifying Respirator } \\
\text { (APR) }\end{array}$ & \$s\$ & \multicolumn{2}{|c|}{ Typically same efficiency of FFP2-FFP3. Filters can usually be replaced [42]]. } & $\begin{array}{l}\text { Fit test is required; } \\
\text { Lung powered with discomfort caused by prolonged } \\
\text { usc; } \\
\text { Negative pressure: if not fitted properly, contaminants } \\
\text { can get into the mask. } \\
\text { May or may not include face shield, which is } \\
\text { recommended for AGPs. }\end{array}$ \\
\hline $\begin{array}{l}\text { Powered Air Purifying } \\
\text { Respirator } \\
\text { (PAPR) }\end{array}$ & sss-ssss & \multicolumn{2}{|c|}{ 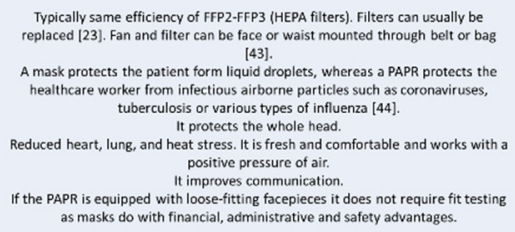 } & $\begin{array}{l}\text { Only workers trained to do so can make repairs or } \\
\text { adjustments to respirators [45]. In case of damage a } \\
\text { new unit is often needed that involves higher costs. }\end{array}$ \\
\hline
\end{tabular}

Novelty: low-cost, easy to make and flat-pack, easy to ship

PAPRs have typically been expensive and not as readily available or as easy to ship to low-resource settings as N95 respirators. Therefore, this paper aims to provide an open-source design for a PAPR prototype which can be made using low cost and off-the-shelf components and materials, with common manufacturing techniques such as laser cutting and 3D printing. The objectives were to make it simple to assemble, easy to clean, flat-pack for easy shipping and functionally comparable to more expensive, commercially available respirators.

\section{METHODS}

The presented prototype respirator is designed to provide protection for healthcare staff against COVID-19 where respiratory protective equipment is not available or prohibitively expensive, or where logistics make single-use items impossible, both for supply and clinical waste handling. The Methods section covers the design requirements, materials and manufacturing techniques used to create this prototype. The Results section provides the designs, with images and links to the CAD files, and the results of testing the prototype. 


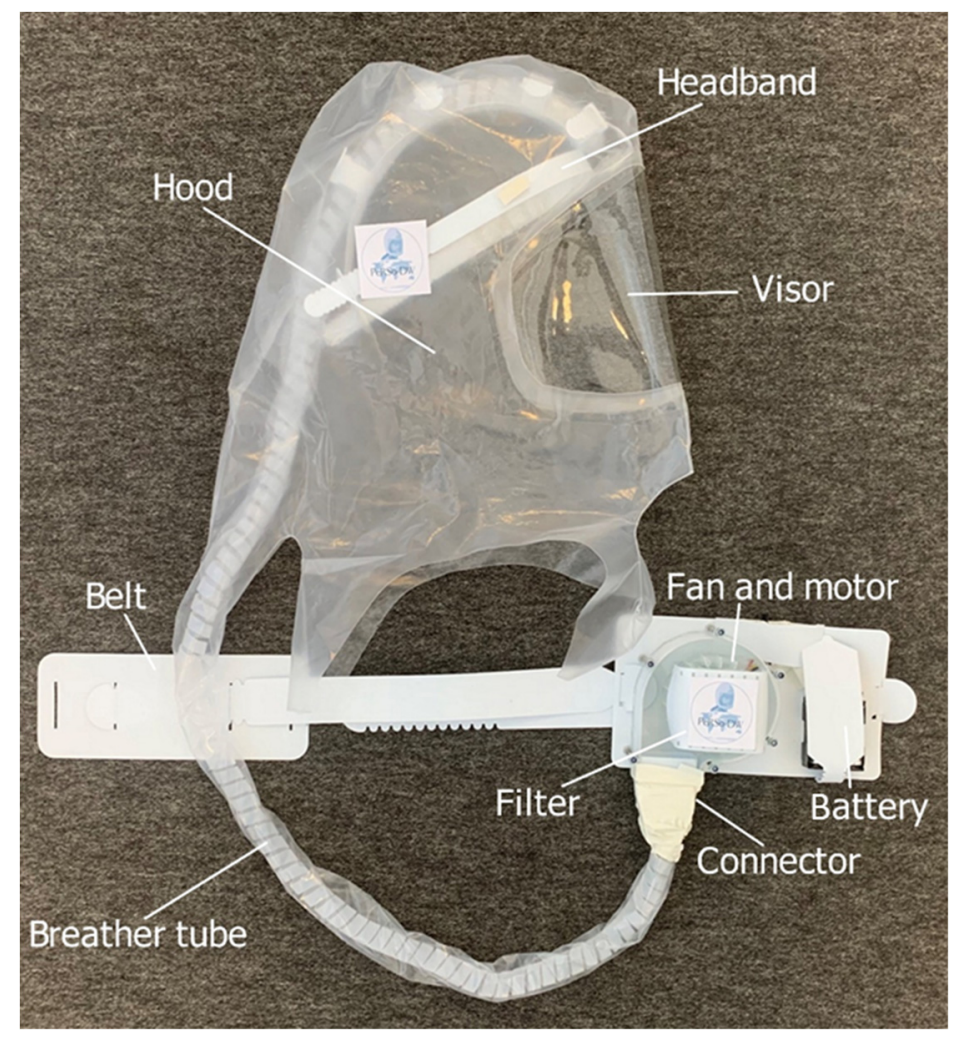

Figure 1 - Flat-pack PeRSo respirator with parts labelled.

The prototype has been divided into 7 parts: the hood and visor; the headband; the breather tube; the centrifugal fan, motor and battery; the filter and filter case; the connector; and the belt that holds the fan and electronics (Figure 1). For each part, the design aimed to meet the following specifications:

- flat or compact, prior to assembly,

- made of easily available, low-cost materials,

- easy to manufacture (using a laser cutter and a 3D printer),

- easy to assemble using common tools such as glue and screwdrivers,

- easy to clean and sterilise after each use,

- durable, efficient and meeting regulatory standards.

CAD software was used to draw designs (find them at the DOI provided above), and the prototype was built using a laser cutter (LS1690 PRO Laser, HPC Laser LTD., UK; native software: Lasercut53) and a 3D printer (Ultimaker S5, Ultimaker).

\section{Specifications for each part:}

\section{Hood}

Design: The hood is designed to go over the whole head, covering the face to protect the face from airborne droplets, splashes, and infection by self-contact with the hands. This design means that the air flowing into the hood creates a positive pressure in the hood that enables the wearer to breathe filtered air easily, without requiring an air-tight seal. This prevents the skin damage that can be caused by pressure or rubbing of a seal against the skin. The design aims to be comfortable, lightweight and sound transmitting, with a clear panel across the whole face so the wearer can see and communicate easily with patients and their family members and carers. It needs to be able to fit 
all face shapes comfortably. The hood also needs to be simple to put on, take off and clean without spreading any virus that is present on the outside surface of it.

Materials, manufacturing and assembly: The materials used are propylene for the hood and transparent Tuftane thermoplastic polyurethane for the visor. A laser cutter is used both to cut the material and fuse the seams. Double sided tape is used to attach the visor to the hood, and Velcro is used to attach the hood to the headband.

\section{Headband}

Design: The headband supports the hood and the breather tube, so they can deliver the filtered air to the face. It also keeps the hood off the face. It needs to be easily adjustable to fit the individual, so that the device is comfortable and stays in place. As part of the headband, a diffuser spreads the flow of the air from the breather tube more evenly across the face.

Materials, manufacturing and assembly: Polypropylene sheet is cut to shape using a laser cutter, and Velcro is used to attach it to the hood. The headband slots together and features adjustable bands for resizing.

\section{Breather tube}

Design: a breather tube is required to transport the filtered air from the fan to the hood, because the weight of the fan and battery is more comfortable to wear on the back or the waist, rather than on the head. It is therefore a requirement that the breather tube is lightweight and flexible, so it does not limit the movements of the wearer.

Materials, manufacturing and assembly: Any type of medical grade tube can be used. In order to have the most flexibility, this prototype breather tube is made out of a common spiral cable tidy and covered in lay-flat polypropylene tubing.

\section{Centrifugal fan, motor and batteries}

Design: The fan consists of impeller plates and blades, along with a case; a brushless motor and case; and screws. Ideally, the battery should enable the device to power for 8 hours, or the standard length of a healthcare worker's shift. However, if the device does not meet this requirement, it is easy to change over the batteries when they are getting low.

Materials, manufacturing and assembly: This prototype uses a drone motor (A2212/6T 2200KV operating voltage: $7.2 \mathrm{~V}$ to $12 \mathrm{~V}$ with no load current: $0.5 \mathrm{~A}$ ). The centrifugal fan is laser cut, and the case for the motor is 3D printed. This prototype uses 6 rechargeable NiMh, $1.2 \mathrm{~V}, 1300 \mathrm{mAh}$ AA batteries (Duracell).

\section{Filter and filter case}

Design: The filter needs to remove virus participles from the air and be easily replaceable if it gets damaged or clogged up. The filter case needs to protect the filter and be easy to clean.

Materials, manufacturing and assembly: This prototype uses a HEPA-FLO NVM-1CH (Numatic International) filter and the filter case is made out of laser cut Perspex, covered in polypropylene for easy cleaning.

\section{Connector}

Design: A connector is required to link the air output from the centrifugal fan to the breather tube. There needs to be an airtight seal at the entrance to the breather tube, so unfiltered air does not enter the breather tube and the filtered air efficiently reaches the hood.

Materials, manufacturing and assembly: The connectors are laser cut out of polypropylene and sealed with duct tape. 


\section{Belt}

Design: The belt is designed to hold the fan and the electronics in an ergonomic manner suitable for healthcare workers. It is designed to be easy to access the internal parts, easy to clean, and have size-adjustable straps for the waist.

Materials, manufacturing and assembly: The belt is made out of laser cut out of polypropylene and the pieces slot together.

\section{Testing the prototype}

Initial testing was performed on the prototype to test that its battery life was suitable for a full health-worker shift length, the airflow rate was fast enough for the wearer to breathe easily and to have no $\mathrm{CO}_{2}$ build-up in the hood, and to check the sealing of components.

\section{a) Battery life}

A commercial floating ball flowmeter was used to assess the flow rate of the fabricated fan in outlet after mounting it with the HEPA filter material. A small section of hose $(26 \mathrm{~mm}$ internal diameter, Part no. CUXS10, Griflex, Birmingham, UK) was used to connect the fan to the flowmeter. Flow rate was kept at $165 \mathrm{~L} / \mathrm{min}$ [46], increasing the voltage over time to ensure ideal flow rate would reach the hood through the breather tube.

\section{b) Flowmeter test}

An anemometer (Kestrel 5200 Professional Weather Meter 0852LHVG, Kestrel Meters, USA) was employed to test the flow rate of the assembled fan with the electronics mentioned above.

\section{c) Nebuliser bitter test to check sealing of components}

Commercially available nebuliser and test solutions (FT-30 \& FT-32 solution and spray apparatus, 3M UK, St Paul, MN, USA) were employed to ensure acceptable positive pressure inside hood, particulate efficacy of filter, and sealing around assembly. The bitter solution was sprayed 30 times directly in front of the visor while the users wearing the respirators performed simple actions such as breathing normally, breathing heavily, tilting the head left/right and up/down, talking and bending. During these tests, a user wearing the respirator should not taste or smell the atomised bitter solution. As a positive control, the solution was sprayed close to the users' face to ensure sensibility to the compound.

\section{RESULTS}

\section{Hood}

The hood polypropylene was sourced from standard rubble bags $(508 \mathrm{~mm} \times 762 \mathrm{~mm}$ from Polystar Plastics Ltd, UK). The transparent rubble bag (effectively two layers of polypropylene sheets sealed at the long edges and at one short edge) was placed on the bed of the laser cutter and the laser was focussed at a height of $40 \mathrm{~mm}$ (this value depends on the machine employed). This height, which changes according to the different machines available, is optimal for a well-focused laser which can cut the chosen material, in specific areas. Figure 2 shows the hood and visor pattern. The black lines were cut with a speed of $150 \%$ and a power of $50 \%$ (Figure 2.A, black lines). After this step, the laser cutter was also used to cut and seal the blue lines on the hood. The laser was moved out of focus at a height of $60 \mathrm{~mm}$ above the rubble bag. The cut and seal process was achieved with a speed of $20 \%$ and a power of $25 \%$ (Figure 2.A, blue lines).

To make the visor, a $0.5 \mathrm{~mm}$ thick sheet of transparent Tuftane thermoplastic polyurethane (TPU) was laser cut to fit the region of hood corresponding to the face. TPU was cut with a speed of $50 \%$ and a power of $30 \%$ (Figure 2.B). Then the Tuftane visor was attached internally to the rubble bag hood with double sided tape to ensure the sealing. Figure 2.C shows the assembled hood and Figure $\mathrm{S} 1$ shows the laser cutting process of the hood. 
A.

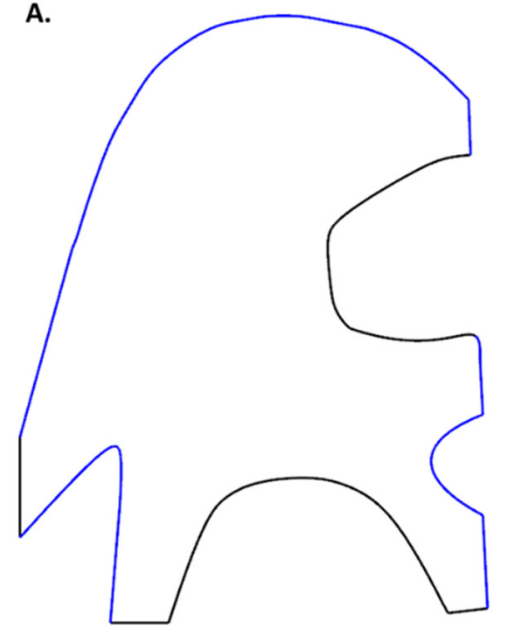

B.

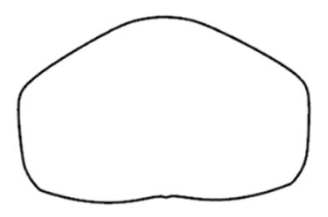

$80 \mathrm{~mm}$

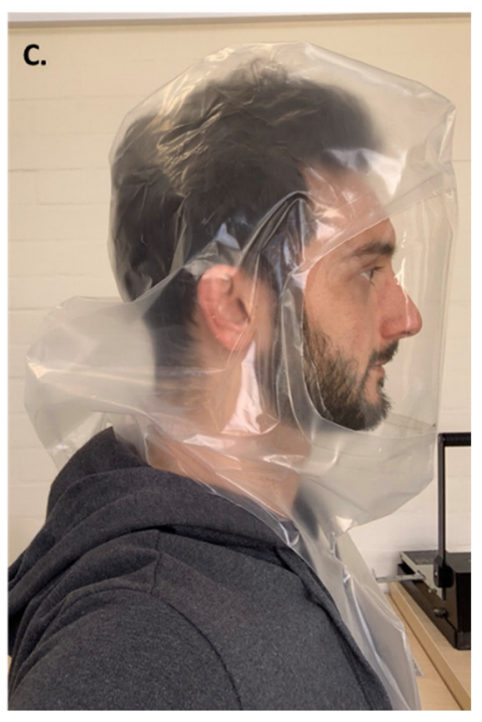

Figure 2 - Hood from rubble bag. A) side design of hood to be laser cut from rubble bag. Black lines are to be cut, and leave open features or edges, whereas blue lines are to be cut and sealed, creating a seam; B) visor design to be cut and taped to the upper opening of the hood; C) complete fabricated hood design with visor.

\section{Headband}

The headband was fabricated by laser cutting of $0.75 \mathrm{~mm}$ thick polypropylene sheet. The headband was designed to be easy to assemble without requiring further parts or tools. It is made of three parts: (i) a strip which when assembled goes on the sides of the head and comprises an air diffuser on the forehead. The ends are designed as a series of inverted Us which then lock into a back plate (Figure 3.A); (ii) a strip to hold the headgear from sliding down and to hold the breather tube in position (Figure 3.B); and (iii) a back plate which has a rectangular shape with small slots that can fit the strips in order to adjust the size of the headband on the sides and on the top of the head (Figure 3.C). This allows for the headband to be easily adjusted to head sizes spanning from $480 \mathrm{~mm}$ to 640 $\mathrm{mm}$ in circumference (or different sizes when editing the design provided). The part was laser cut with a speed of $75 \%$, a power of $100 \%$ and a corner power of $30 \%$.

Figure 3.D shows the complete headband. Figure $S 2$ and Video 1 show the assembling process.
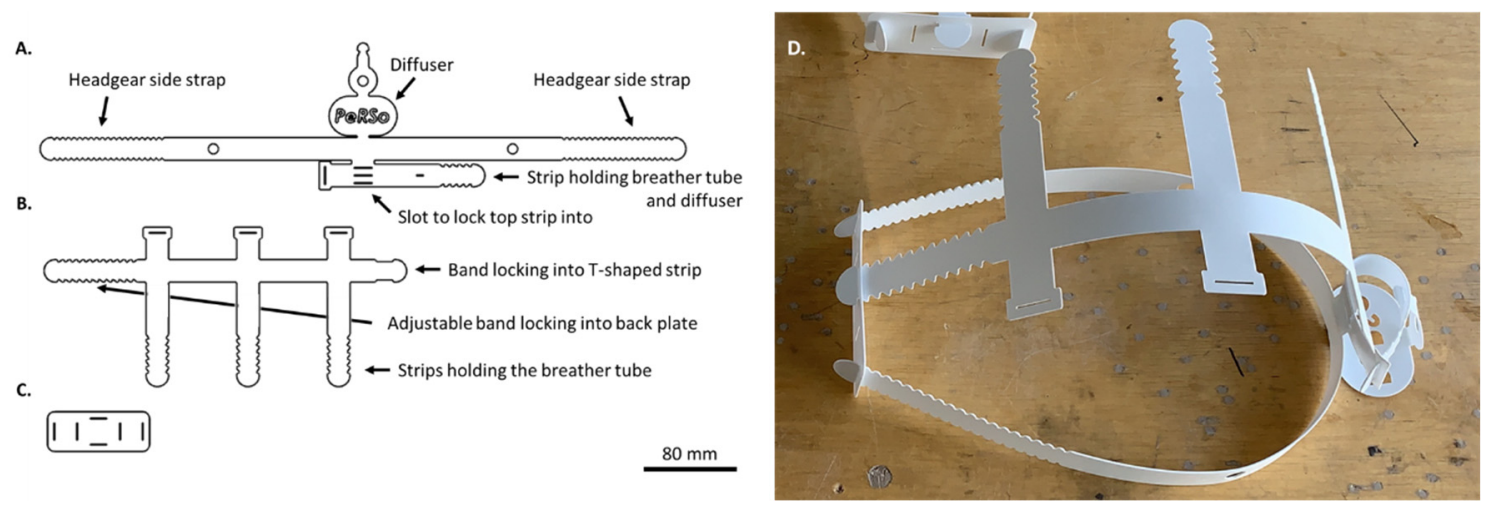

Figure 3 - Headband to hold hood and breather tube on user's head. A) T-shaped strip that goes around the sides of the head, contains the PeRSo shaped diffuser and joins with (B) and (C) to form the complete headband; B) top strip to hold the breather tube that connects to (A) through (C);C) back plate with vertical slots for (A) and horizontal slots for (B); $D)$ the assembled headband. 


\section{Breather tube}

The breather tube has an external diameter of $30 \mathrm{~mm}$ and internal diameter of $28 \mathrm{~mm}$. It was made out of common spiral cable tidy ( $30 \mathrm{~mm}$ external diameter) and covered in lay flat tubing (polypropylene, 2" $\times 500$ gauge) (see Figure 4). The tube length can be tailored to the height of the user; however, a length of $120 \mathrm{~mm}$ is appropriate for most users ranging from $1.60 \mathrm{~m}$ to $1.90 \mathrm{~m}$ in height. This results in a very deformable and light breather tube that allows for air flow to travel from fan to hood.
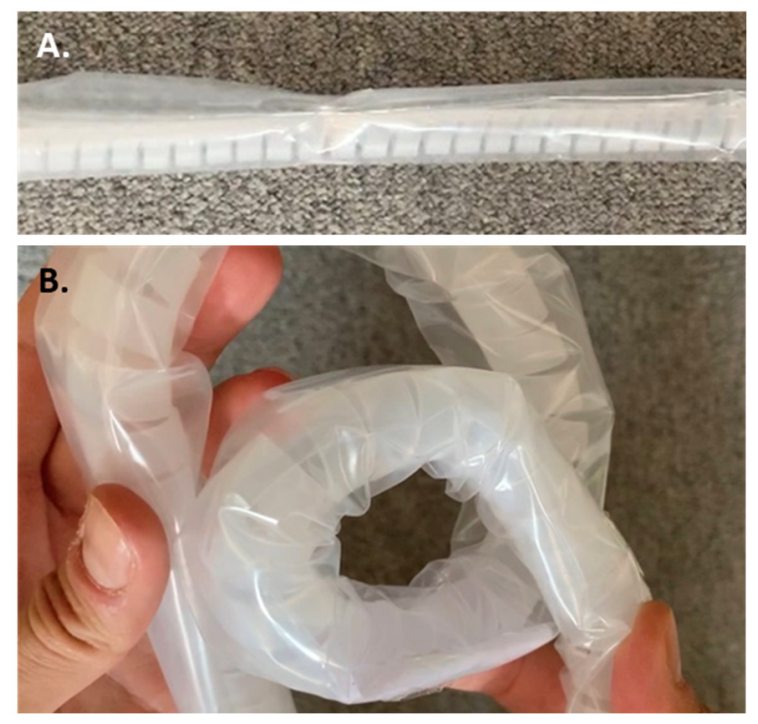

Figure 4-The breather tube, made of spiral cable tidy and covered in polypropylene lay-flat tubing.

The breather tube was glued in position at the top part of the headband by the headgear side straps and its outlet flows onto the diffuser at the forehead. Then the headband was attached inside the hood using Velcro straps. Figure $\mathrm{S} 3$-left shows the upper part of the respirator which consists of hood, headband and breather tube. The total weight of the upper part was $150 \mathrm{~g}$.

\section{Centrifugal fan and motor}

The centrifugal fan has the following components:

1. Fan case: made of laser cut Perspex ( $3 \mathrm{~mm}$ thick) (Figure $5 . \mathrm{A}$ ) and laser cut polypropylene (0.75 mm thick) (Figure 5.D)

2. Impeller plates: laser cut out of Perspex ( $3 \mathrm{~mm}$ thick) (Figure 5.B)

3. Impeller blades (18): laser cut out of polypropylene ( $0.75 \mathrm{~mm}$ thick) (Figure $5 . \mathrm{C}$ )

4. Brushless motor (off-the-shelf drone motor) and 3D printed case (Figure 5.G-frame).

5. M4 screws for fan case, M3 screws for impeller, M3 screws for motor case.

The laser cutter settings for the Perspex were: speed $40 \%$, power $100 \%$ (cut twice); for engraving (red in Figure $5 . A$ ) offset $0.2 \mathrm{~mm}$ (x6) speed 50\%, power 20\%. Laser cutter settings for polypropylene: speed $75 \%$, power $100 \%$, corner power $30 \%$. 
A.

B.
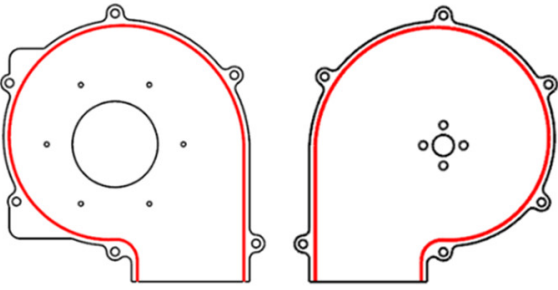

c.

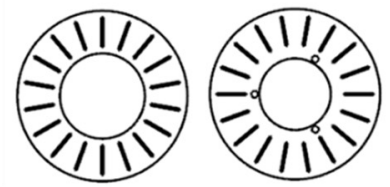

D.

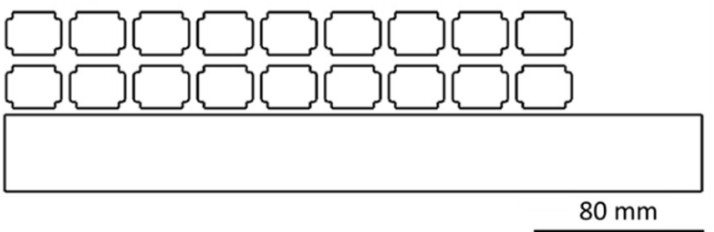

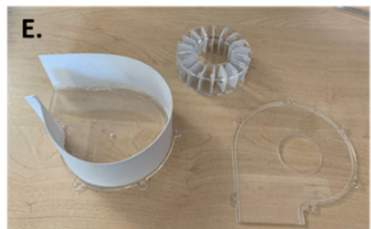

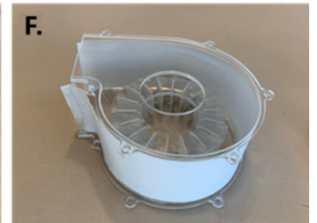

G.
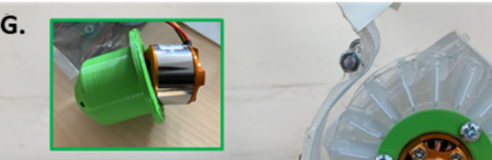

ó

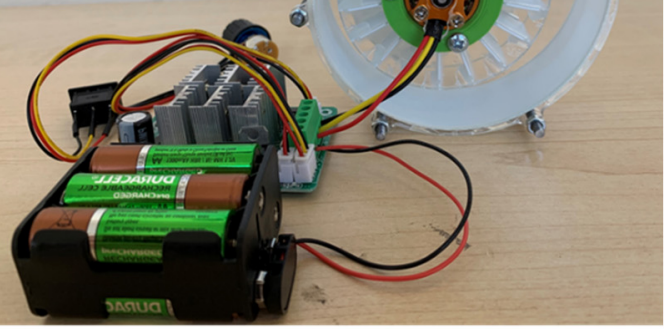

Figure 5 - Centrifugal fan assembly with off-the-shelf drone motor. A) top plate (left) with opening for air intake, and back plate (right) with holes for motor screws and spindle. Red lines show the path in which the wall strip in (D) locks; B) impeller plates with slots for blades, and holes to hold motor case so that the impeller spins with the motor; $C$ ) impeller blades; D) strip locking into red path in (A) which encloses the impeller; (E) laser-cut parts for centrifugal fan are (F) assembled using hot glue. The top fan plate is attached to the back assembly using screws for easy access to drone motor; (G) assembly of centrifugal fan with drone motor, battery pack and controller connected to it. The controller has a I/O switch and a wheel to control the voltage supplied to the fan. The green frame shows the assembly of the drone motor in the $3 D$ printed holder which connects to the impeller to allow rotation.

The brushless drone motor (A2212/6T 2200KV - operating voltage: $7.2 \mathrm{~V}$ to $12 \mathrm{~V}$ with no load current: $0.5 \mathrm{~A}$ ), was connected to a speed regulator controller (Part ID: Keensonvqgo3z9sw, CS Priority Industrial Ltd, China) which is also connected to $6 \mathrm{AA}$ batteries ( $7.2 \mathrm{~V}$ total). The controller has a potentiometer that can be turned to adjust the voltage provided to the motor and hence the flow rate going into the hood. In order to connect the motor to the fan case, a motor case was designed, and 3D printed. The 3D printing settings were: infill $60 \%$, layer height $0.2 \mathrm{~mm}$, adhesion, no support.

Video 2 shows the assembling of the centrifugal fan.

However, 3D printing can be avoided if the drone motor is equipped with an integrated screw on top of the spindle that can be anchored through a nut to the impeller. This would result in the whole respirator being manufactured just by laser cutting, which makes the process much faster than 3D printing.

Once the fan parts were laser cut, the fan back plate was hot glued to the wall strip, and the impeller blades were glued to the plates (Figure 5.E-F). The top casing plate was connected to the back assembly by $6 \mathrm{M} 3$ screws for easy access to the motor. The drone motor was placed into the 3D printed holder (green frame in Figure 5.G) and this was secured to the back impeller plate, whereas the drone motor was attached to the fan back plate by M2 screws (supplied with the motor) going into the drone motor body. Then, the motor was connected to the controller and the battery pack.

\section{Filter and filter case}

The filter case was mounted outside the fan so that the air that is drawn first through the filter for purification, then into the fan, and blown into the breather tube and into the hood. The filter case 
was made out of laser cut Perspex (Figure 6.A) and covered with laser cut $0.75 \mathrm{~mm}$ thick polypropylene (Figure 6.B) that can be easily cleaned.

Filter medium of choice was HEPA material from vacuum bags (HEPA-FLO NVM-1CH, Numatic International), which was wetted before being laser cut (speed 100\%, power 30\%) (Figure 6.C).

A.

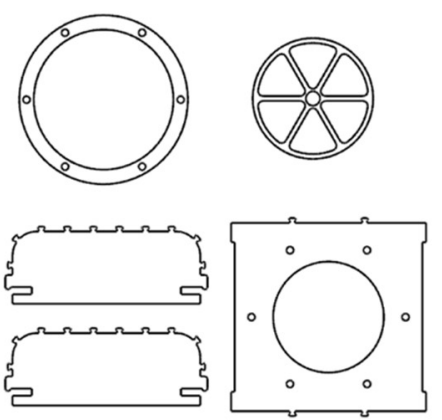

B.

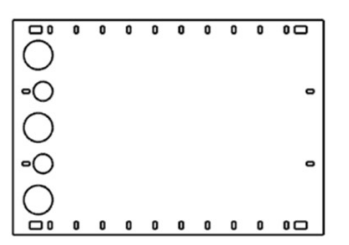

c.

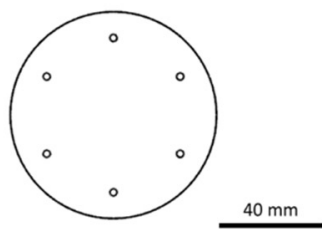

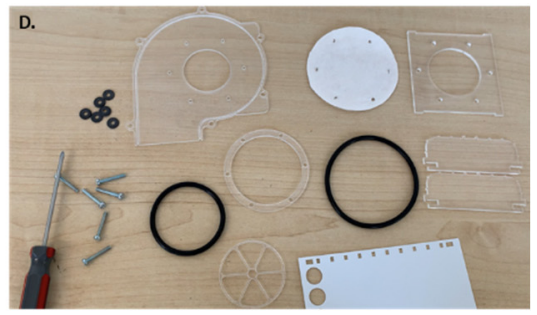



Figure 6 - Filter case with HEPA filter. A) clockwise from top left: i) ring to hold filter material, ii) ring to support filter material, iii) back plate to hold filter in place, iv) side plates for filter cartridge; $B$ ) cover for filter cartridge for easy cleaning and protection from spills and droplets; C) cut design for filter material of choice; D) components for filter case. Rubber O-rings and rubber washers were used to ensure sealing of the assembly; E) assembled filter case on the left with cover on the right.

The filter case was assembled to ensure sealing of all components (Figure 6.D) so that the air flowing into the fan would be completely filtered before going into the breather tube. A large O-ring was positioned on top of the fan case, then the next layer was the cut-out Perspex ring with six holes for screws inside, followed by a smaller O-ring and the back plate to support the filter. Then the filter material and the plate for the fan cover were placed on top of the assembly and rubber washers were aligned to the 6 holes. These were held in place by 6 screws (left in Figure 6.E).

For the fan cover, the side Perspex plates were then attached to the polypropylene sheet through pins and slots respectively (right in Figure 6.E). The assembled filter cover then slides into the backfilter cover on the first assembly and it is held in place securely by two pairs of pins and slots in the back plate and the filter cover respectively. This ensures that the filter case is well sealed and allows for easy cleaning of the fan system.

Video 3 shows the assembling of the filter case.

\section{Connector}

A connector was designed to attach the fan and the hood to the breather tube. The connector was laser cut out of polypropylene (speed $75 \%$, power $100 \%$, corner power $30 \%$ ) and has a pyramidal shape connecting the outlet of the fan to the inlet of the breather tube (Figure 7.A). The design allows for connection to different size tubes. The assembled connector is shown in Figure 7.B and it was later sealed with duct tape to the fan case and the breather tube to make the system airtight. 

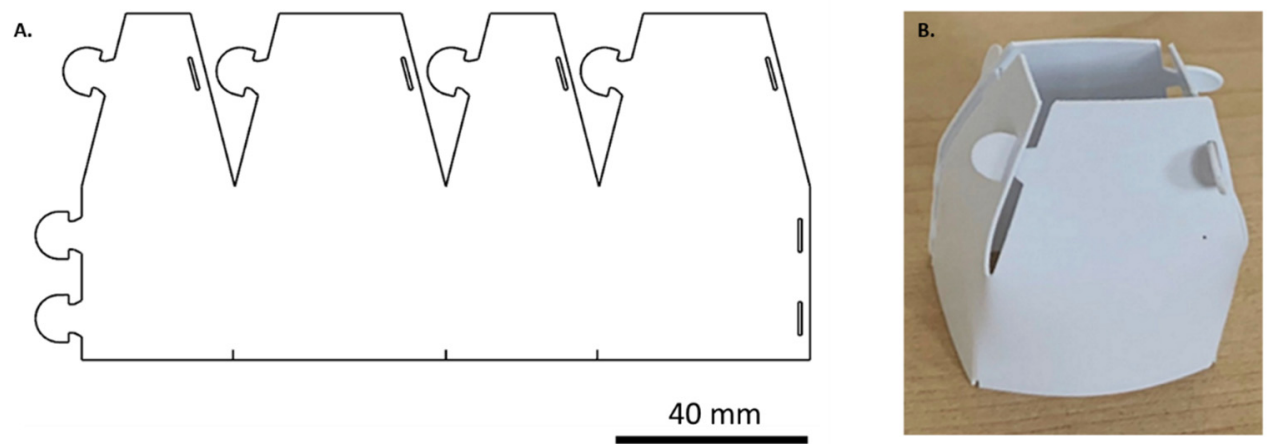

Figure 7-Connector for breather tube to centrifugal fan. A) Design; B) Laser-cut and assembled connector.

\section{Belt}

The belt was made out of laser cut $0.75 \mathrm{~mm}$ thick polypropylene (speed: $75 \%$, power: $100 \%$, corner power: $30 \%$ ). The belt has a large plate to support (i) the fan, through 6 screws which are used to hold the fan case (Figure 8.A), (ii) the controller, that is mounted at the upper edge of the belt for easy access, and (iii) the battery pack, mounted in a pocket (Figure 8.B). The straps (Figure 8.D) interlock in a frontal plate with slots and can be adjusted according to user's waist (Figure 8.C).

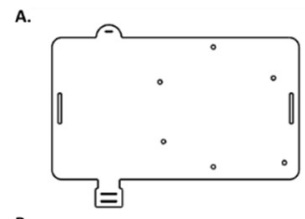

B.
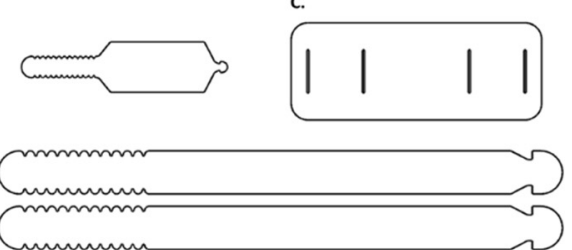

$80 \mathrm{~mm}$



Figure 8-Belt to hold the blower units with the electronics. A) belt back plate holding battery pack on the left and centrifugal fan on the right via the 6 screws in the fan case; B) strip holding battery pack. This connects to the horizontal slots in (A); C) front plate with slots to lock the waist strips (D) with Ushaped features to allow for different lengths to be chosen according to user's waistline; E) fabricated belt.

The belt was assembled as shown in Figure 8.E. The blower unit containing the electronics (battery pack and controller) and the fan connected to the belt is shown in Figure $\mathrm{S} 3$-right. The controller allows the voltage supplied to the fan to be adjusted, by rotating the switch, which modifies the flow rate. The total weight of the blower unit is $600 \mathrm{~g}$.

\subsubsection{Final prototype}

Figure 1 shows the final prototype, connecting the hood-headband-breather tube upper assembly to the connector-blower unit-electronics-belt lower assembly. The prototype has a clean, simple and light appearance. Such a design, as showed previously by Elkington et al. 2020 [32] results in a friendly look, helping people to see each other's face and communicate easily. 
The total cost of the materials used in this prototype was $\sim f 50$, which makes it a low-cost device. The total weight is $750 \mathrm{~g}$ and it occupies a volume of $300 \mathrm{~mm}$ (L) $\times 300 \mathrm{~mm}$ (W) $\times 8 \mathrm{~mm}$ (D) when assembled and stored.

Video 4 shows the demonstration of a fully developed prototype.

\subsection{Flow rate, battery life and sealing tests}

The flow rate of the drone motor and flat-pack fan was tested at $7.2 \mathrm{~V}$ and at the fan outlet a flow rate of $190 \mathrm{~L} / \mathrm{min}$ was recorded. Pre-use testing with an anemometer prototype may be useful to ensure that the flow is sufficient for the usage. Flow may vary because of battery degradation or tube length adjustment, therefore checking the flow before setting up the device is recommended.

The performance of the assembled blower unit in terms of battery life was tested by using a floating ball flowmeter. The motor was switched on and the fan was controlled manually so that the flowmeter indicated $165 \mathrm{~L} / \mathrm{min}$, for as long as the battery charge allowed. Using $6 \mathrm{NiMh}, 1.2 \mathrm{~V}, 1300$ mAh AA batteries, the fan was able to deliver $165 \mathrm{~L} / \mathrm{min}$ for 2 hours and 45 minutes. Increasing the total current supplied (i.e. the number of batteries connected in parallel) will consequently enhance the battery life of the device. Using a power supply such as a power bank or battery packs equipped with LEDs may be helpful to see the charge level and understand the remaining run time of the power supply.

Additionally, the full prototype was fit tested by using a bitter solution atomised via a nebuliser. Users could not taste the solution when wearing the respirators and while performing different tasks, meaning that the hood properly seals the head. As a positive control, users could taste the bitter solution when not wearing the flat-pack respirator.

\section{DISCUSSION}

The results demonstrate the feasibility of designing and building a low-cost PAPR using off-the-shelf components, laser cutting and 3D printing. The paper provides detailed information on how to build this simple, flat-pack design. Initial tests suggest that this design has comparable efficacy with more expensive, commercially available respirators. Moreover, the materials used are selected for easy cleaning and sterilisation after every use. The filter case is also designed so that it can work as a cartridge, and different filter media can be integrated in the respirator.

With SARS-CoV-2 spreading rapidly in low-resource settings, healthcare systems are strained in both staffing and infrastructure. The chronic supply issues related to disposable PPE are acutely compounded by the coronavirus pandemic and associated economic disruption. Increased mortality amongst healthcare workers has already been observed in high income countries [3][4], so it is vital that PPE is adequate and available to protect the healthcare workers in low-resourced settings, where there are shortages of healthcare workers. Recent UK data also indicates the greatest risk of infection in Black and Asian healthcare workers, irrespective of their working location, but this may due to the types of employment they hold, their greater reliance on public transport and living in multigenerational or large households, compared to their white British counterparts, rather than a genetic disposition [47]. It is important to remember to provide protection for all people who work in healthcare settings, including cleaners and administrators, not only clinicians, and to consider how to protect people on their commute to work.

Proper protocols need to be put in place for wearing, removing and cleaning the respirators as well, because the virus that the respirator protects workers from is likely to land on the outside surface of 
the respirator, so needs to be removed with care to stop the virus from spreading to the wearer's hands and face [32].

PAPRs represent an alternative strategy to standard filtering face covering PPE that may have particular benefits for low-resource settings. Firstly, one reusable PAPR device can replace the months-long requirement of facemask respirators, visors and caps or, realistically, avoid the risks of re-using these single-use devices out of necessity. Secondly, healthcare staff will be more efficient, able to move from patient to patient without repetitively changing their PPE. Thirdly, as the whole face can be seen in a PAPR, communication is easier than with single-use face coverings, and finally, PAPRs may be more comfortable than a tight-fitting face covering in hot climates because of the cool air flow reducing sweat build-up, and avoidance of loading the face's vulnerable soft tissues.

However, the PAPR design has some limitations. It can be more restricting of movement, due to being bulkier than a single-use face covering, and because it covers the ears and has a fan, it can be harder to hear through it and to use a stethoscope. It is therefore useful to prioritise a low-noise fan, to direct the flow of air away from the ears, and to use materials that are not noise-insulating. The addition of sensors and warning alarms to alert the wearer if the battery is low or the flow rate from the fan has decreased beyond the recommended levels, can be useful for making sure the healthcare workers remain protected throughout the day.

Often commercially available PAPRs are much higher specification than required for healthcare use, as they are designed for industries such as welding. Therefore, we developed a cheap and simple respirator. The components are deliberately suitable for mass production and often repurposed from other widely available sources. The designs are presented in full, to support in-country manufacture. They are available open source, with no intellectual property protection and all data on the manufacture process is published online. In the present work, we have avoided direct reverse-engineering, and are not aware of any intellectual property conflicts. However, we have not undertaken a full international freedom to operate search, so we recommend that manufacturers using this design run an independent intellectual property search to make sure there are no infringements. In the case of infringements, governments may be able to invoke legislation like Crown Use Exemption to authorise use of IP without the owner's agreement, or the World Trade Organisation's Agreement on Trade Related Aspects of Intellectual Property Rights, but individuals should be aware of the legal risks of infringement.

Local checks and regulatory approval will be needed to confirm the efficacy of respirators after manufacture, such as checking for ingress around the filter using the spray test solutions. We will provide technical updates on our website and aim to develop this as a community of practice, showing different potential manufacturing solutions suitable for different geographic settings. We propose that widespread manufacturing and use of PAPRs may reduce the impact of the SARS-CoV-2 pandemic, both short and long term, by enabling more effective healthcare provision and keeping staff safe to care for others.

\section{Acknowledgements}

We would like to acknowledge the support of the PeRSo-DW team members: Ric Gillams, Alexandra Mant, David Marshall, Hywel Morgan, Philippa Reed, Clint Styles and Liku Tezera; and the Institute for Life Sciences (IfLS) for initiating our collaboration. 
We thank Chris Malcolm from the Design Workshop at the University of Southampton for his support with the equipment needed for the fabrication of the prototype, and Dan Edmunds from Permali Gloucester Ltd for supplying Tuftane material used for the visors.

We gratefully acknowledge support from the University of Southampton Strategic Development Fund, which is funded by the UK Government as part of the Global Challenges Research Fund (GCRF), and projects that seconded staff to this work including MRC (MR/P023754/1 (PTE)), EPSRC (EP/L025035/1 (ADG) and EP/R014213/1 (ASD, LED)), and donations to the Southampton Coronavirus Response Fund. We thank Action 4 Equality (Scotland) Limited ( $\mathrm{Dr}$ Alison Steele and $\mathrm{Mr}$ Stefan Cross, QC) for the gift provided to support this research.

Additional data has been made openly available from the University of Southampton repository at https://doi.org/10.5258/SOTON/D1582. 


\section{References}

[1] B. Ivorra, M. R. Ferrández, M. Vela-Pérez, and A. M. Ramos, "Mathematical modeling of the spread of the coronavirus disease 2019 (COVID-19) taking into account the undetected infections. The case of China," Commun. Nonlinear Sci. Numer. Simul., vol. 88, p. 105303, 2020, doi: 10.1016/j.cnsns.2020.105303.

[2] "who-director-general-s-opening-remarks-at-the-media-briefing-on-covid-19---11-march2020 @ www.who.int." [Online]. Available: https://www.who.int/dg/speeches/detail/whodirector-general-s-opening-remarks-at-the-media-briefing-on-covid-19---11-march-2020.

[3] M. A. Mamun and I. Ullah, "COVID-19 suicides in Pakistan, dying off not COVID-19 fear but poverty? - The forthcoming economic challenges for a developing country," Brain. Behav. Immun., vol. 87, pp. 163-166, 2020, doi: 10.1016/j.bbi.2020.05.028.

[4] P. G. T. Walker et al., "The impact of COVID-19 and strategies for mitigation and suppression in low- And middle-income countries," Science (80-. )., 2020, doi: 10.1126/science.abc0035.

[5] World Health Organization, "Health Workforce Requirements for Universal Health Coverage and the Sustainable Development Goals," Hum. Resour. Heal. Obs. Ser., no. 17, pp. 1-40, 2016, doi: ISBN 9789241511407.

[6] T. P. Velavan and C. G. Meyer, "The COVID-19 epidemic," Trop. Med. Int. Heal., vol. 25, no. 3, pp. 278-280, 2020, doi: 10.1111/tmi.13383.

[7] L. Rodriguez, "5 Reasons COVID-19 Will Impact the Fight to End Extreme Poverty," Global Citizen, Apr. 2020.

[8] E. B. Ing, Q. A. Xu, A. Salimi, and N. Torun, "Physician deaths from corona virus (COVID-19) disease," Occup. Med. (Lond)., vol. 70, no. 5, pp. 370-374, 2020, doi: 10.1093/occmed/kqaa088.

[9] C. Smith, "The structural vulnerability of healthcare workers during COVID-19: Observations on the social context of risk and the equitable distribution of resources," Soc. Sci. Med., vol. $258,2020$.

[10] Y. Liu et al., "Viral dynamics in mild and severe cases of COVID-19," Lancet Infect. Dis., vol. 20, no. 6, pp. 656-657, 2020, doi: 10.1016/S1473-3099(20)30232-2.

[11] Y. Bai et al., "Presumed Asymptomatic Carrier Transmission of COVID-19," JAMA, vol. 323, no. 14, p. 1406, Apr. 2020, doi: 10.1001/jama.2020.2565.

[12] A. N. Desai and P. Patel, "Stopping the Spread of COVID-19," JAMA - J. Am. Med. Assoc., vol. 323 , no. 15, p. 1516, 2020, doi: 10.1001/jama.2020.4269.

[13] N. Chen et al., "Epidemiological and clinical characteristics of 99 cases of 2019 novel coronavirus pneumonia in Wuhan, China: a descriptive study," Lancet, vol. 395, no. 10223, pp. 507-513, 2020, doi: 10.1016/S0140-6736(20)30211-7.

[14] World Health Organization, "Modes of transmission of virus causing COVID-19 : implications for IPC precaution recommendations," 2020. doi: 10.1056/NEJMoa2001316.5.

[15] N. Van Doremalen et al., "Aerosol and surface stability of SARS-CoV-2 as compared with SARS-CoV-1," N. Engl. J. Med., vol. 382, pp. 1564-1567, 2020, doi: 10.1056/NEJMc2004973.

[16] B. E. Young et al., "Epidemiologic Features and Clinical Course of Patients Infected with SARSCoV-2 in Singapore," JAMA - J. Am. Med. Assoc., vol. 323, no. 15, pp. 1488-1494, 2020, doi: 10.1001/jama.2020.3204.

[17] Y. Liu et al., "Aerodynamic analysis of SARS-CoV-2 in two Wuhan hospitals," Nature, vol. 582, pp. 557-560, 2020, doi: 10.1038/s41586-020-2271-3. 
[18] J. P. O. Li, D. S. C. Lam, Y. Chen, and D. S. W. Ting, "Novel Coronavirus disease 2019 (COVID19): The importance of recognising possible early ocular manifestation and using protective eyewear," Br. J. Ophthalmol., vol. 104, no. 3, pp. 297-298, 2020, doi: 10.1136/bjophthalmol2020-315994.

[19] S. Kumwenda, "Challenges to Hygiene Improvement in Developing Countries," in The Relevance of Hygiene to Health in Developing Countries, 2019.

[20] S. M. Moghadas et al., "Projecting hospital utilization during the COVID-19 outbreaks in the United States," Proc. Natl. Acad. Sci. U. S. A., vol. 117, no. 16, pp. 9122-9126, 2020, doi: 10.1073/pnas.2004064117.

[21] V. Roberts, "To PAPR or not to PAPR?," Can. J. Respir. Ther., vol. 50, no. 3, pp. 87-90, 2014, [Online]. Available: https://www.ncbi.nlm.nih.gov/pmc/articles/PMC4456839/pdf/cjrt-5087.pdf.

[22] L. Amendola, M. T. Saurini, F. Di Girolamo, and F. Arduini, "A rapid screening method for testing the efficiency of masks in breaking down aerosols," Microchem. J., vol. 157, no. May, p. 104928, 2020, doi: 10.1016/j.microc.2020.104928.

[23] R. A. Howard, G. W. Lathrop, and N. Powell, "Sterile field contamination from powered airpurifying respirators (PAPRs) versus contamination from surgical masks," Am. J. Infect. Control, vol. 48, no. 2, pp. 153-156, 2020, doi: 10.1016/j.ajic.2019.08.009.

[24] T. S. Alderman, M. A. Stiegel, R. A. Estes, W. R. Thomann, and G. D. Sempowski, "Field-testing method for loose-fitting powered air-purifying respirators equipped with HEPA filters," Appl. Biosaf., vol. 21, no. 2, pp. 71-78, 2016, doi: 10.1177/1535676016651249.

[25] J. Wong et al., "Preparing for a COVID-19 pandemic: a review of operating room outbreak response measures in a large tertiary hospital in Singapore," Can. J. Anesth., vol. 67, no. 6, pp. 732-745, 2020, doi: 10.1007/s12630-020-01620-9.

[26] G. A. Brat, S. Hersey, K. Chhabra, A. Gupta, and J. Scott, "Protecting Surgical Teams During the COVID-19 Outbreak," Ann. Surg., no. 617, p. 1, 2020, doi: 10.1097/sla.0000000000003926.

[27] E. L. Daugherty, "Health care worker protection in mass casualty respiratory failure: Infection control, decontamination, and personal protective equipment," Respir. Care, vol. 53, no. 2, pp. 201-212, 2008.

[28] C. M. Clase et al., "Cloth Masks May Prevent Transmission of COVID-19: An Evidence-Based, Risk-Based Approach," Ann. Intern. Med., 2020, doi: 10.7326/m20-2567.

[29] O. Aydin, B. Emon, S. Cheng, L. Hong, L. P. Chamorro, and M. T. A. Saif, "Performance of fabrics for home-made masks against the spread of COVID-19 through droplets: A quantitative mechanistic study," Extrem. Mech. Lett., vol. 40, 2020, doi: 10.1016/j.eml.2020.100924.

[30] V. C. Tam, S. Y. Tam, W. K. Poon, H. K. W. Law, and S. W. Lee, "A reality check on the use of face masks during the COVID-19 outbreak in Hong Kong," EClinicalMedicine, vol. 22, no. April, p. 100356, 2020, doi: 10.1016/j.eclinm.2020.100356.

[31] Q. Chen, B. Lim, S. Ong, W. Wong, and Y. Kong, "Rapid ramp-up of powered air-purifying respirator (PAPR) training for infection prevention and control during the COVID-19 pandemic," no. January, 2020, [Online]. Available: https://www.ncbi.nlm.nih.gov/pmc/articles/PMC7158777/pdf/main.pdf.

[32] P. Elkington et al., "A Personal Respirator Specification for Health-care Workers Treating COVID-19 ( PeRSo )," vol. 19, 2020.

[33] A. Repici et al., "Coronavirus (COVID-19) outbreak: what the department of endoscopy should know," Gastrointest. Endosc., vol. 92, no. 1, pp. 192-197, 2020, doi: 
10.1016/j.gie.2020.03.019.

[34] X. He, T. Reponen, R. T. McKay, and S. A. Grinshpun, "Effect of particle size on the performance of an N95 filtering facepiece respirator and a surgical mask at various breathing conditions," Aerosol Sci. Technol., vol. 47, no. 11, pp. 1180-1187, 2013, doi: 10.1080/02786826.2013.829209.

[35] J. Smereka, K. Ruetzler, L. Szarpak, K. J. Filipiak, and M. Jaguszewski, "Role of mask/respirator protection against SARS-CoV-2," Anesth. Analg., vol. XXX, no. Xxx, pp. 1-2, 2020, doi: 10.1213/ANE.0000000000004873.

[36] T. M. Cook, "Personal protective equipment during the coronavirus disease (COVID) 2019 pandemic - a narrative review," Anaesthesia, vol. 75, no. 7, pp. 920-927, 2020, doi: 10.1111/anae.15071.

[37] M. Assadi et al., "Key elements of preparedness for pandemic coronavirus disease 2019 (COVID-19) in nuclear medicine units," Eur. J. Nucl. Med. Mol. Imaging, vol. 2019, pp. 17791786, 2020, doi: 10.1007/s00259-020-04780-4.

[38] D. Lepelletier et al., "Respiratory protective equipment at work: good practices for filtering facepiece (FFP) mask," Eur. J. Clin. Microbiol. Infect. Dis., vol. 38, no. 11, pp. 2193-2195, 2019, doi: 10.1007/s10096-019-03642-8.

[39] S. A. Lee, D. C. Hwang, H. Y. Li, C. F. Tsai, C. W. Chen, and J. K. Chen, "Particle size-selective assessment of protection of european standard FFP respirators and surgical masks against particles-tested with human subjects," J. Healthc. Eng., vol. 2016, 2016, doi: 10.1155/2016/8572493.

[40] J. E. Coia et al., "Guidance on the use of respiratory and facial protection equipment," J. Hosp. Infect., vol. 85, no. 3, pp. 170-182, 2013, doi: 10.1016/j.jhin.2013.06.020.

[41] V. Puro, N. Magnavita, and G. Ippolito, "SARS and masks," J. Hosp. Infect., vol. 56, no. 1, pp. 73-74, Jan. 2004, doi: 10.1016/j.jhin.2003.09.010.

[42] A. Chakladar, P. O. Beaumont, and D. R. Uncles, "Respirator fit-testing - will we pass the test?," Crit. Care, vol. 13, no. 5, p. 2009, 2009, doi: 10.1186/cc8031.

[43] R. J. Roberge, A. Coca, W. J. Williams, J. B. Powell, and A. J. Palmiero, "Reusable elastomeric air-purifying respirators: Physiologic impact on health care workers," Am. J. Infect. Control, vol. 38, no. 5, pp. 381-386, 2010, doi: 10.1016/j.ajic.2009.11.006.

[44] 3M.CO.UK, "Powered Air \& Supplied Air Respirators," [Online]. Available: https://www.3m.co.uk/3M/en_GB/company-uk/3m-products/ /All-3M-

Products/Safety/Worker-Health-Safety/Powered-Supplied-Air-

Respirators/?N=5002385+8709322+8711017+8711405+8720547\& rt=r3.

[45] W. Bischoff, J. Turner, G. B. Russell, M. Blevins, and J. Stehle, "Evaluation of a Novel Powered Air-purifying respirator (PAPR) vs. a N95 Respirator Mask for the Protection Against Influenza in a Human Exposure Model," Open Forum Infect. Dis., vol. 4, no. suppl_1, pp. S168-S168, 2017, doi: 10.1093/ofid/ofx163.298.

[46] J. Zhu, X. He, M. S. Bergman, S. Guffey, A. D. Nimbarte, and Z. Zhuang, "A pilot study of minimum operational flow for loose-fitting powered air-purifying respirators used in healthcare cleaning services," J. Occup. Environ. Hyg., vol. 16, no. 7, pp. 440-445, Jul. 2019, doi: 10.1080/15459624.2019.1605241.

[47] Z. Haque, L. Becares, and N. Treloar, "A Runnymede Trust and ICM Survey Over-Exposed and Under-Protected," 2020. 
PeRSo is a prototype and this Specification is for information purposes only. It has not been properly tested for safety, efficacy or durability whether for single, multiple or repeated use. We have not obtained CE certification, regulatory approval or validated PeRSo units as a whole, and/or the constituent components, so as to comply with the relevant Standard for personal respiratory equipment. Final quality assured PeRSo units' intended use is in a health care setting. PeRSos should not be used in an industrial or manufacturing environment, drilling, welding, close to flames, chemicals or fumes or otherwise where existing, if similar looking, products would be more suitable.

Individuals should not rely on the information in this specification and attempt to self-assemble their own PeRSo. They should seek to purchase a quality assured PeRSo device from a reputable manufacturer who provides safety warnings, use instructions, gives product liability assurances, guarantees and has effective product liability insurance in place. There is an increased risk of contamination by individuals untrained in specialist donning, doffing and cleaning protocols. Any individual's use of self-made PeRSo is entirely at their own risk.

Manufacturers taking forward the production of PeRSo do so at their own risk. Manufacturers will need to develop their own manufacturing specification and undertake or obtain the necessary quality assurance, regulatory approvals, CE marking and/or compliance to the appropriate Standard prior to supplying or selling any PeRSo or releasing onto the market. Manufacturers should also undertake their own intellectual property freedom to operate checks and have in place appropriate product liability insurance.

Organisations using PeRSo or related devices should establish careful protocols for "donning", "doffing" and cleaning to prevent these devices being a source of contamination to individuals.

\section{DISCLAIMER}

Except as represented here the Specification for the PeRSo Prototype is provided "AS IS". The University of Southampton gives no assurance, guarantee or warranty, express or implied, that any PeRSo device manufactured or assembled following this specification: will be safe to use; will be of merchantable quality; will be fit for any particular purpose; will protect individuals from contracting COVID-19 or any other infectious disease or virus whilst using it; or that individuals using it will not suffer personal injury or death. The University of Southampton does not warrant or in any way assert that the manufacture, sale or use of PeRSos will not infringe the intellectual property of any other person or organization. The University of Southampton hereby disclaims any and all warranties, express or implied, and excludes, to the maximum extent permitted by law, any and all liability for any loss, harm, damage, injury or death suffered by any person caused by, or resulting from, their reliance on the information, designs and Specifications provided. 


\section{Supplementary material}
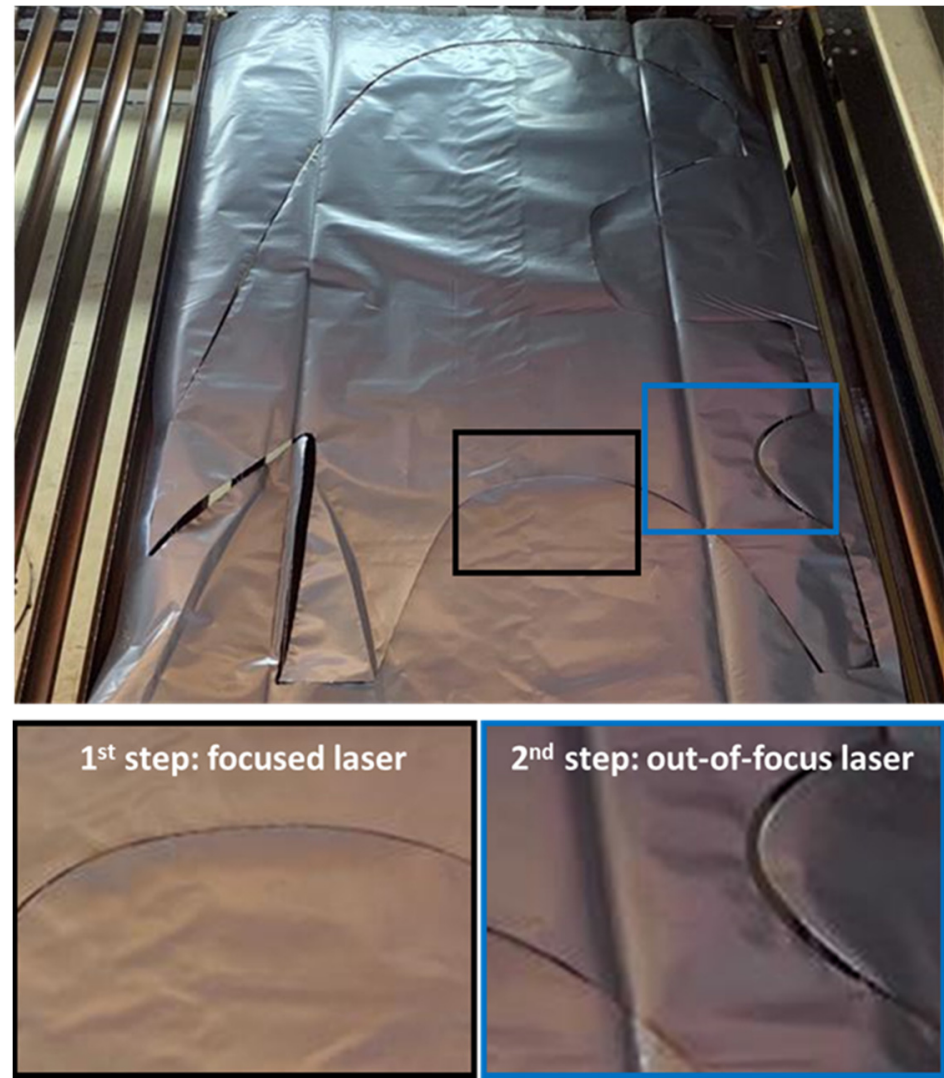

Figure S 1 - The hood was fabricated out of rubble bags laser cut following a 2-step procedure, where the laser was focused to cut some features (black frame) such as the visor, the shoulder and the tube areas, and was moved out of focus to cut-seal other features (blue frame) such as the top, the back and the front under the chin areas. On the right, complete hood design.

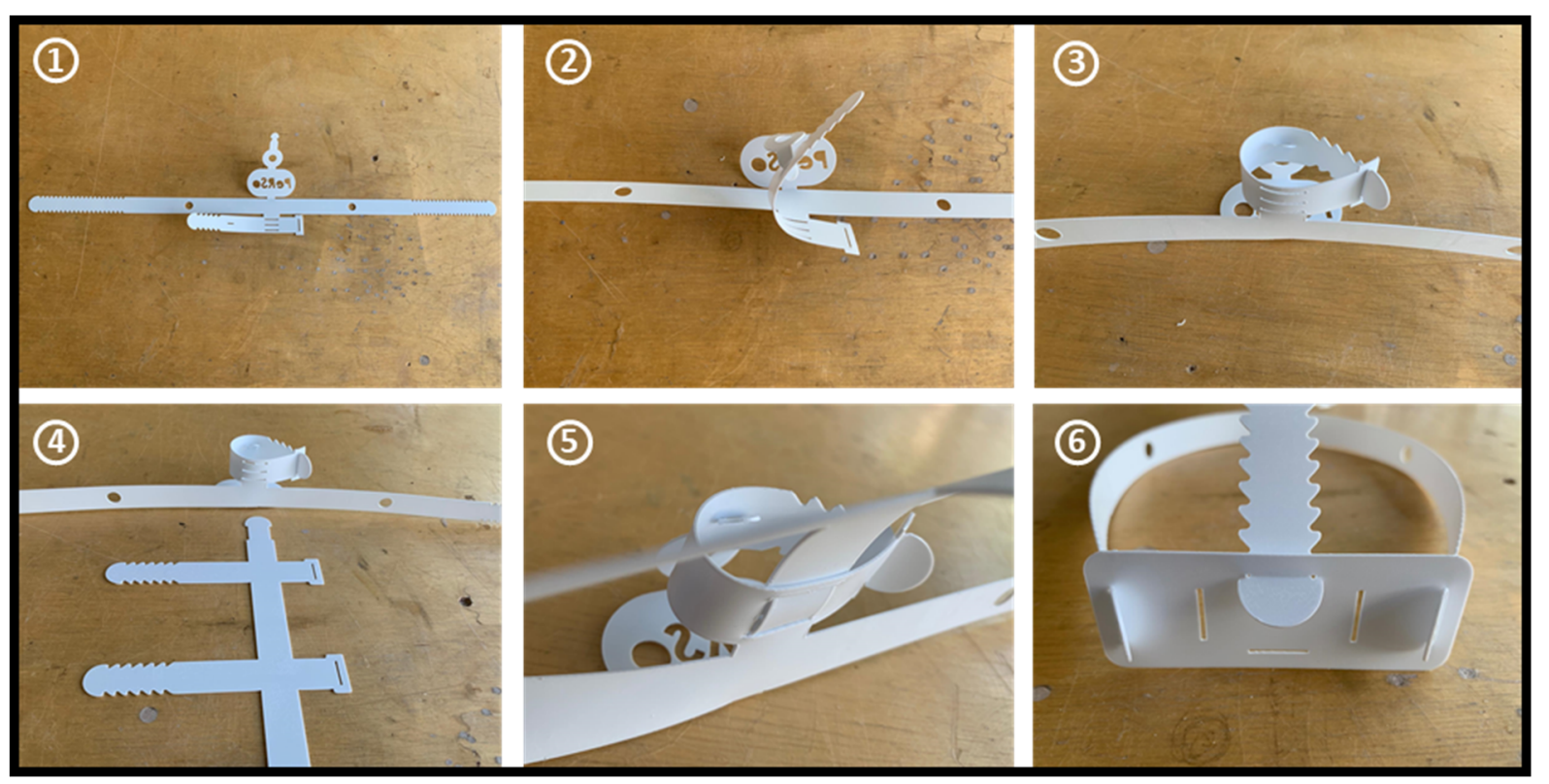

Figure S 2 -Assembling process from 2D sheet to 3D fully functional headband. 



Figure S 3 - Assembly of respirator. Left) Upper part - assembly of hood, headband and breather tube (top-left) and fit test of assembly on user; Right) Lower part - assembly of fan to breather tube connector and laser-cut belt to hold the blower unit and the electronics.

\section{Additional material available at https://doi.org/10.5258/SOTON/D1582}

\section{Videos of assembly:}

Video 1 - Headband.mp4

Video 2 - Centrifugal fan with drone motor.mp4

Video 3 - Filter case.mp4

Video 4 - Demonstrating PeRSo Flat-pack Respirator.mp4

\section{CAD files for each part in PeRSo_Flat_pack_CAD_files.zip:}

1 - Hood and visor.dxf

2 - Headband.dxf

3 - Centrifugal fan.dxf

4 - Drone motor mount.stl

5 - Filter case.dxf

6 - Connector.dxf

7 - Belt.dxf 